\title{
Ethnobotanical study of subtropical hills of Darazinda, Takht-e-Suleman range F.R D.I. Khan, Pakistan
}

\author{
Ulfat Samreen ${ }^{1}$, Muhammad Ibrar ${ }^{1}$, Lal badshah ${ }^{1}$, Shahida Naveed ${ }^{2}$, \\ $\operatorname{Imran}^{3 *}$ and Inayat $\mathrm{Khatak}^{3}$ \\ 1. Department of Botany, University of Peshawar, Pakistan \\ 2. Government Girls Degree College Karak, Pakistan \\ 3. Department of Agronomy, University of Agriculture, Peshawar, Pakistan \\ *Corresponding author's email: imranagrarian@aup.edu.pk
}

Citation

Ulfat Samreen, Muhammad Ibrar, Lalbadshah, Shahida Naveed, Imran and Inayat Khatak. Ethnobotanical study of subtropical hills of Darazinda, Takht-e-Suleman range F.R D.I. Khan, Pakistan. Pure and Applied Biology. Vol. 5, Issue 1, 2016, pp149-164. http://dx.doi.org/10.19045/bspab.2016.50020

\begin{tabular}{llll}
\hline \hline Received: 21/10/2015 & Revised: 12/01/2016 & Accepted: 24/01/2016 & Online First: 01/02/2016 \\
\hline \hline
\end{tabular}

\section{Abstract}

The present study contributes to ethnobotanical information of Darazinda (Frontier Region) D.I. Khan, Khyber Pakhtunkhawa, Pakistan. A Total of 198 plant species belonging to 62 families were recorded, in which Poaceae (35), Asteraceae (20), Solanaceae (12), Brassicaeae and Papilionaceae each 8, Amaranthaceae 6, Boraginaceae, Chenopodiaceae, Cucurbitaceae and Euphorbiaceae 5 each, Asclapidiaceae, Polygonaceae 4 each, Malvaceae, Mimisiaceae, Apiaceae, Cyperaceae, Rhamnaceae, Myretaceae, Moraceae, Verbanaceae, Zygophyllaceae, Scorphulariaceae and Laminaceae 3 each were recorded. The other families possessed 2 or fewer species. Locally these plants are used for agriculture applications (2\%), fodder (65\%), as a fuel $(15 \%)$, for making furniture (4\%), fruit yielding (6\%), as medicinal (55\%), as timber (5\%), as vegetable (13\%) and fencing plants (4\%).

Key words: Ethnobotany; Darazinda; Medicinal plant; Vegetable; Timber; Fencing plant;

Fodder; F.R. D.I. Khan

\section{Introduction}

Darazinda F.R. D.I. Khan is a small Frontier Region of Pakistan. It is also known as Largha Shirani. The area is located between North latitude $31-30^{\circ}$ and $31-34^{\circ}$ and West longitudes $69.55^{\circ}$ and $70.24^{\circ}$. Total area of this region is 3,229 square kilometers. On the North, it is bounded by South Waziristan Agency, on the east by Kulachi, on the South by Musa Khel and Dera Ghazi Khan (Punjab) and on the West by Zhob (Baluchistan) (Figure 1). In this tribal area, there are no urban localities. Darazinda and
Daraban (an adjacent area) are Persian words and are linked to Dara Shikoh, one of the sons of Mughal king Shah Jahan. "Daraban" means Dara's forest, because Dara used to visit the forests of the area for hunting, while "Darazinda" means prison of Dara, where Dara had established a prison. The Western part of this area is composed of Koh-e-Suleman Mountains, while on the eastern side, at lower altitude lies areas like Bargholay, Angharghara, Pasta, Spin ghar and Sur ghar hills. In the Western range a number of peaks of over 2,750 meter are 
present. The highest peak is Takht-eSulemanis $(3,441$ meters $)$. The eastern hills have an average height of $700 \mathrm{~m}$.

Ethnobotanical survey helps to open new avenues for research on scientific times to explore new drugs for treatment of various ailments. Ethnobotanic knowledge has been acquired by local people from centuries of indigenous knowledge by hit and trail method and it is passed from generation to generation. This knowledge provides the starting point to investigate a plant for possible therapeutic effect on scientific basis. Rich phyto diversity of Darazinda consists of large number of plants species, majority of which are used medicinally by the local people who have very limited modern medical facilities. Majority of the population of this area depends upon plant resources as no other jobs are available in the area. Rearing of cattle is good source of food and income of local inhabitants. Wood is used for fuel and the people are dependent on forest resources for first hand money. Grazing of cattles, sheeps, goats, camels, cows are also prominent in this area. Different workers have carried out studies of plant resources of different areas of Pakistan e.g. Sher and Hussain [1] conducted ethnobotanical survey on the medicinally and economically important plants of Malam Jabba valley, District Swat and reported 50 species of plants belonging to 33 families as ethno botanically important Saima et al. [2] collected 167 plants belonging to 65 families from national park of Ayubia, Abbottabad. Fazal et al. [3] collected 211 species of 66 families from District Haripur, Nasrullah et al., [4] reported 67 plants species, belonging to 39 families of Jandool valley, Dir lower, Barkatullah and Ibrar [5] described 169 species belonging to 76 families of Malakand Pass Hills, District Malakand,
Marwat et al., [6] reported 11 wild edible fruit plants species belonging to 8 families from Dera Ismail Khan District, Naveed et al., [7] reported 110 species belonging to 51 families from Shamshokii District Karak., Haq et al. [8] communicated ethno botanical uses of medicinal plants of Allai valley (Western Himalaya Pakistan) and reported that 172 taxa were locally used for various medicinal purposes; Alamgeer et al. [9] evaluated and documented ethnomedicinal knowledge of Alladand Dehri, Tehsil Batkhela, District Malakand and reported 92 plant species for cure of various diseases, Khan and Hussain [10] documented 161 multi-purpose species in Tehsil Takhti -eNasrat, Ahmad and Habib [11] collected 59 Medicinal Plants of Himalaya Region, Dawarian Village, Neelum Valley, Azad Jammu and Kashmir, Hussain et al., [12] documented 69 plant species belonging 29 families and reported plant cover, density and frequency of plants from Chakwal. Arshad et al., [13] Studied vegetation, frequency, value index, density and cover of plants of Cholistan desert, No such ethnobotanical work has been reported from this remote and neglected area of Darazinda and present study is carried out to document the ethnobotanical knowledge of this area.

\section{Materials and method}

Frequent visits of the research area at various seasons were carried during 20132014 in order to collect plants and get ethnobotanical information from knowledgeable people and elders of the area by a questionnaire. Identification of plants was carried out with the help of Flora of Pakistan $[14,15]$, and from the herbarium of Botany Department, University of Peshawar, Pakistan. The identified plants were given voucher numbers and deposited in the Botany department herbarium, University of Peshawar for ready reference. 


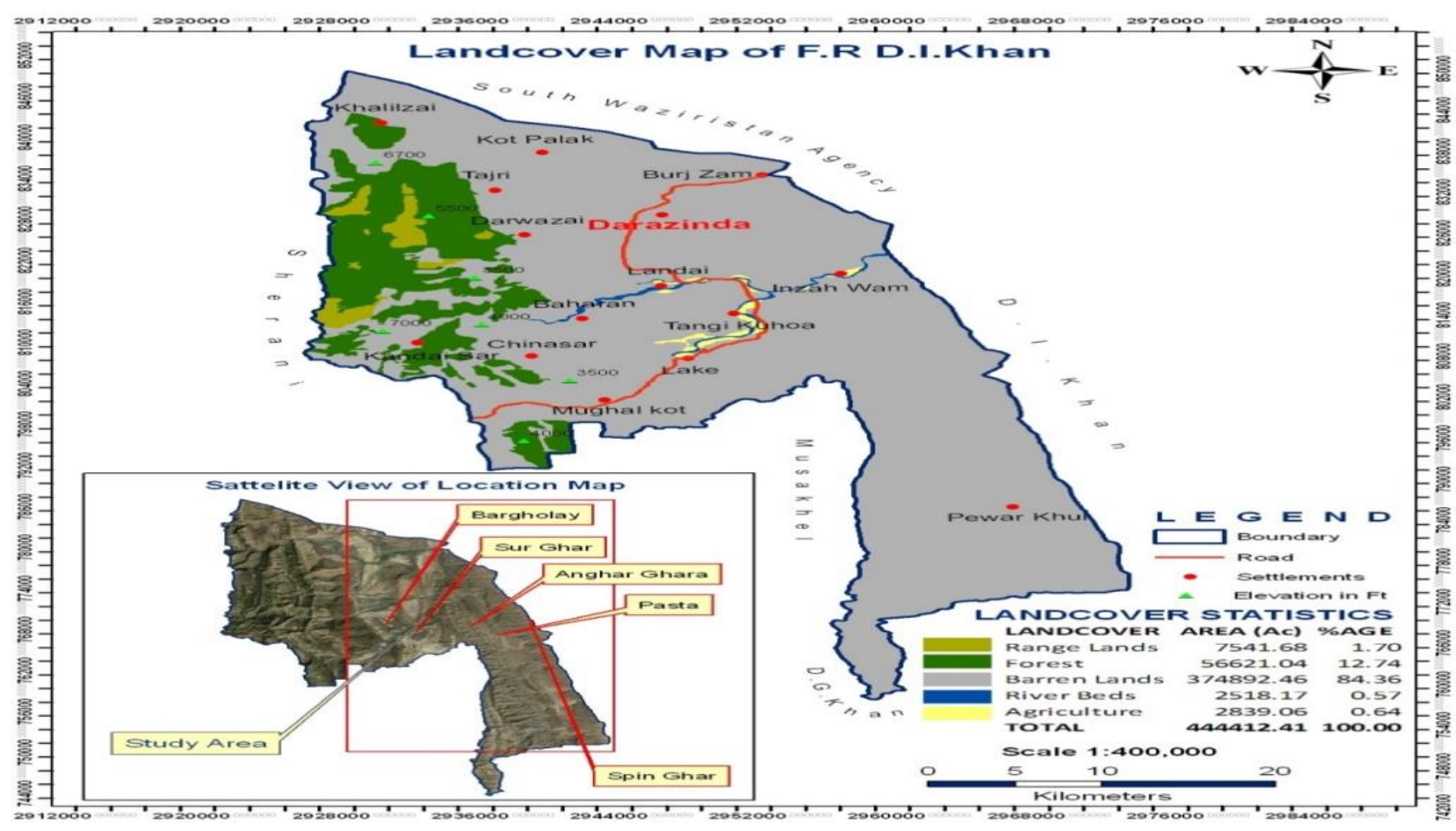

Figure 1. Map of Darazinda F.R. D.I. Khan

\section{Results and discussion}

In the present work a total of 203 plant species have been reported which were mainly used as fodder, medicinal, fuel, fencing, and vegetable and for agriculture tools making. In Figure 2 the ethnobotanical profile of the various plants of the area has been represented graphically. Most of the plants $(65 \%)$ of the area are used as a fodder followed by the medicinal plants (55\%), which are used by the local inhabitants as home remedies for their therapeutically active constituents. Nearly15 \% plant species are used by the locals as fuel wood and $13 \%$ plants as vegetables. Fruit and timber producing trees are $6 \%$ and $5 \%$, respectively. $4 \%$ plants are used for furniture making while $3.5 \%$ plants are for fencing. Only a smaller number of plants $(2 \%)$ are used for agriculture tools making. (Figure 3 to Figure 8) is the good illustration of the various uses of different plants in daily life of the area inhabitants.

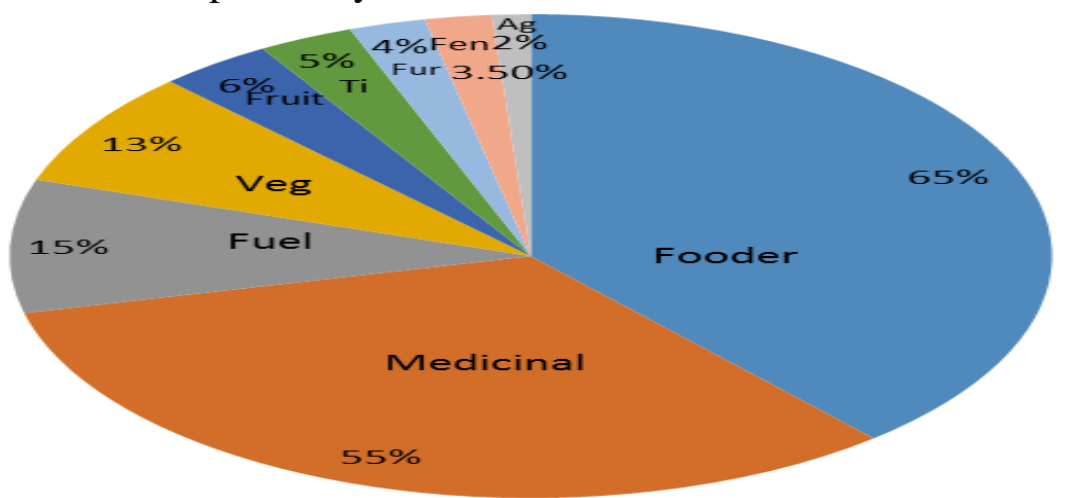

Figure 2. Graphical representation of ethnobotanical profile 


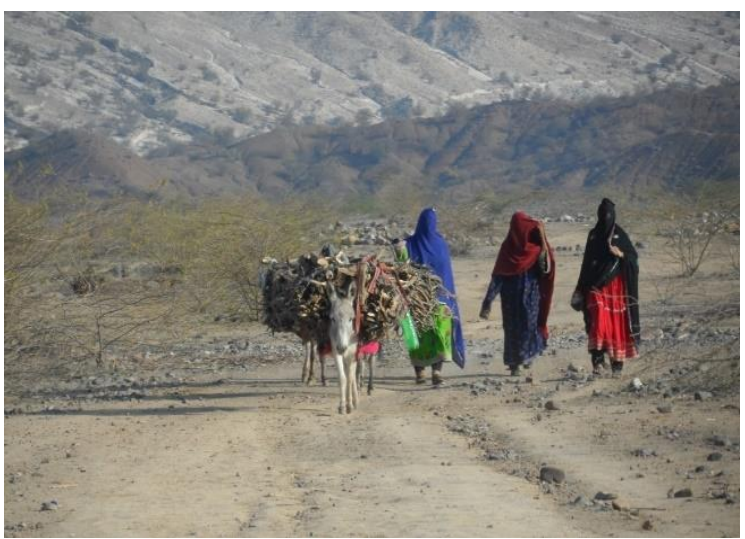

Figure 3. Female carrying wood for fuel

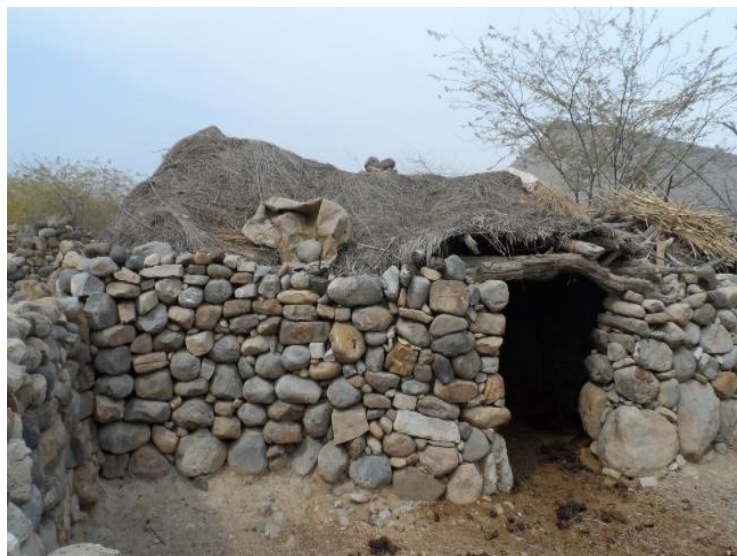

Figure 5. Plants used for thatching

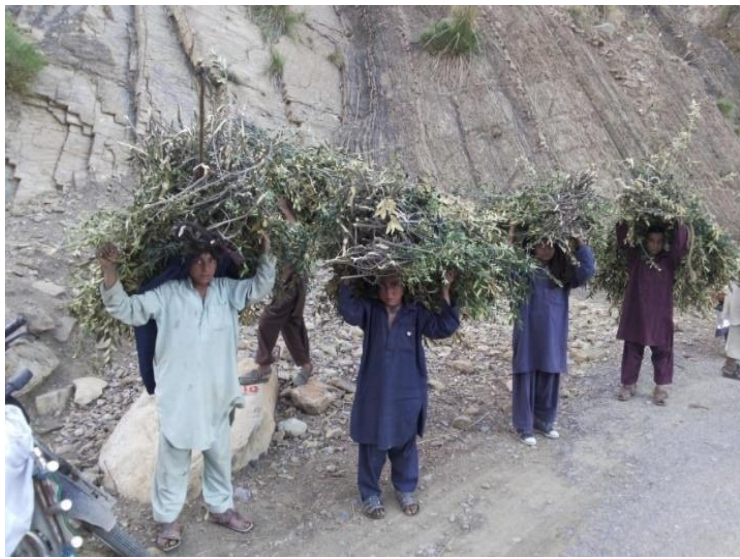

Figure 7. Plants to be used for multipurpose

\section{Fodder utilization}

The results are given in table 1 , which show that a $129(65 \%)$ were used as fodder. Acacia modesta, Achrechne racemosa, Albizia lebbeck, Apluda mutica, Aristida

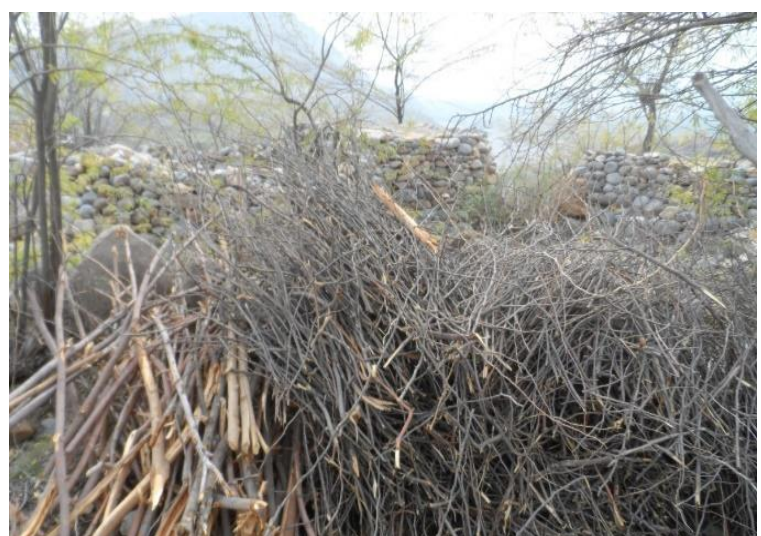

Figure 4. Wood used as fencing as well as fuel

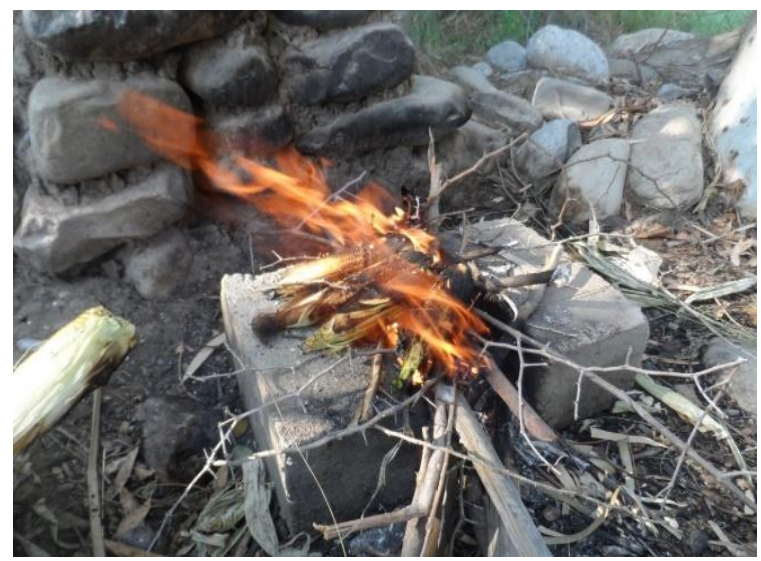

Figure 6. Fuel wood

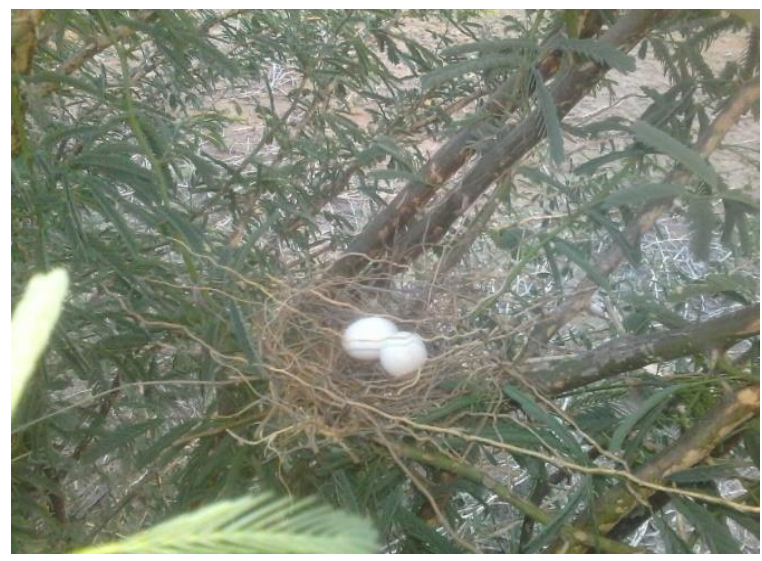

Figure 8. Plants used for nesting

adscensionis, Argyrolobium roseum, Astragalus psilocentros, Bromus japonica, Chenopodium album, Chenopodium murale, Convunvulus arvensis, Cymbopogon jwarancusa, Cynodon dactylon, 
Dichanthium annulatum, Echinochloa colona, Eragrostis minor, Hordeum vulgare, Iphiona grantoides, Lactuca serriola, Lepidium draba, Lolium temulentum, Morus alba, Pennisetum oriantale., Phalaris minor, Plantago lanceolata, Saccharum bengalensis, Rhazya stricta, Salvadora persica, Sonchus asper, Stellaria media, Taraxacum officinale, Tribulus terrestris, Zizyphus mauritiana, Zizyphus oxyphylla were used as fodder by local cattles (Goat, sheep, cow and camel), which caffer their meat and dairy product needs. These cattles are also source of income by selling out them and their milk and wool. Similar results were shown by Ibrar et al. [16] reported 37 forage species from Ranyal hill, Shangla District. Dilshad et al. [17] investigated 25 fodder species similar to this study which used for diseases cure of cattle. Sardar and Khan [18] also reported many species used as fodder. Barkatullah et al. [19] recorded 18 forage species from Charkotli Hills of District Batkhela. Sher et al. [20] studied 66 plant species used as fodder from Chagharzai Valley, Buner District, Pakistan.

\section{Medicinal utilization}

Result showed that 108 (55\%) plants were used medicinally like Acacia nilotica, Acacia modesta, Achyranthus biclentata, Achyranthus aspera, Agaricus campestris, Albizia lebbeck, Allium cepa, Allium sativum, Amaranthus viridis, Astragalus psilocentros, Artemisia scoparaia, Calligonum polygonoides, Caralluma tuberculata, Cannabis sativus, Capparis spinosa, Carthamus oxycantha, Capsicum annum, Chenopodium album, Cordia maxia, Coriandrium sativum, Cucumis sativus, Cucurbita maxima, Cucurbita pepo, Cuscuta reflexa, Dalbergia sissoo, Datura alba, Dodonaea viscosa, Eucalyptus globules, Euphorbia prostate, Fagonia cretica, Foeniculum valgare, Helianthus annus, Peganum harmala, Pennisetum oriantale, Phoenix dactylifera, Portulaca quardrifida, Raphanus sativus, Salvadora persica, Tamarix aphylla, Withania coagulans, Zizyphus oxyphylla, Zizyphus nummularia were locally used as medicinal plants. Fagonia cretica is widely used to cure various diseases including skin diseases, while Salvadora persicais used for cureness of female diseases. Medicinal uses of other plants are given in table 1. Similar results were obtained by Sher and Hussain [1] they reported 50 medicinal plants species of 30 families from Malam Jaba. Ullah et al. [21] reported traditional 34 medicinal plant species from Darra Adam Khel KPK, Pakistan. Jan et al. [22], Sher et al. [20] and Mahmood et al. [23] also carried out medicinal plants study of different areas of the country. Similar work also reported from Tehsil Karak by Khan et al., [24] and from Tehsil Takht-e-Nasrati by Khan et al., [25], Bonet et al. [26] and Ajaib et al. [27].

\section{Fuel utilization}

The result showed that $30(15 \%)$ plants were used as fuel in this area. Acacia nilotica, Acacia modesta, Albizia lebbeck, Calligonum polygonoides, Cymbopogon jwarancusa, Dalbergia sissoo, Desmostachya bipinnata, Dodonaea viscosa, Eucalyptus globules, Eucalyptus lanceolatus, Melia azedarach, Morus nigra, Periploca aphylla, Phoenix dactylifera, Phylla nodiflora, Tamarix aphylla, Zizyphus oxyphylla, Zizyphus mauritiana, Zizyphus nummulariaetc are used as fuel. Figure 2 showing females of the area carrying wood for fuel). Similar studies were carried out by Sher et al. [20] reported 51 wood species used as fuel from Chagharzai Valley, Buner District, Pakistan. More than $80 \%$ of the hilly area people depend upon the surrounding hills for collection of fuel plants. Deka et al. [28], Ajaib et al. [27] also reported similar results. 
Table 1. Presented plant families/species, fodder, medicinal, fuel, vegetables, fruit yielding, timber, furniture, fencing and agriculture appliances

\begin{tabular}{|c|c|c|c|c|c|c|c|c|c|c|}
\hline $\begin{array}{l}\text { S. } \\
\text { No. }\end{array}$ & Plant families/species & Fodder & Medicinal & $\begin{array}{l}\text { Fuel } \\
\text { (dried } \\
\text { plants) } \\
\end{array}$ & Vegetables & $\begin{array}{l}\text { Fruit } \\
\text { yielding }\end{array}$ & Timber & Furniture & $\begin{array}{l}\text { Fencing } \\
\text { plants }\end{array}$ & $\begin{array}{l}\text { Agriculture } \\
\text { appliances }\end{array}$ \\
\hline \multicolumn{11}{|c|}{$\begin{array}{l}\text { A. PTERIDOPHYTA } \\
\text { 1. Equisetaceae }\end{array}$} \\
\hline 1 & Equisetum arvense $\mathrm{L}$. & + & - & - & - & - & - & - & - & - \\
\hline \multicolumn{11}{|c|}{ 2. Adiantaceae } \\
\hline 2 & Adiantum capillusveneris $L$ & - & - & - & - & - & - & - & - & - \\
\hline \multicolumn{11}{|c|}{$\begin{array}{l}\text { B. Fungi } \\
\text { 3.Agaricaceae }\end{array}$} \\
\hline 3 & Agaricus campestris L. & - & + & - & - & - & - & - & - & - \\
\hline \multicolumn{11}{|c|}{ 4. Helveliaceae } \\
\hline & Morchella esculenta Fries & - & - & - & - & - & - & - & - & - \\
\hline \multicolumn{11}{|c|}{$\begin{array}{l}\text { C. MONOCOTYLEDONS } \\
\text { 5. Alliaceae }\end{array}$} \\
\hline 4 & Allium sativum L. & - & + & - & + & - & - & - & - & - \\
\hline 5 & Allium cepa $\mathrm{L}$. & - & + & - & + & - & - & - & - & - \\
\hline \multicolumn{11}{|c|}{ 6. Arecaceae } \\
\hline 6 & Nannorrphs ritchiana $\mathrm{H}$. Wendl. & + & - & - & - & - & - & - & - & - \\
\hline 7 & Phoenix dactylifera $\mathrm{L}$. & + & + & + & - & + & + & + & + & - \\
\hline \multicolumn{11}{|c|}{ 7. Asparagaceae } \\
\hline 8 & Asparagus gracilis Royle & + & - & - & - & - & - & - & - & - \\
\hline \multicolumn{11}{|c|}{ 8. Cyperaceae } \\
\hline 9 & Cyprus elumoids L. & + & - & - & - & - & - & - & - & - \\
\hline 10 & Cyperus rotundrus & + & - & - & - & - & - & - & - & - \\
\hline 11 & Eleocharis palustris (L.) & + & - & - & - & - & - & - & - & - \\
\hline \multicolumn{11}{|c|}{ 9. Poaceae } \\
\hline 12 & Apluda mutica & + & - & - & - & - & - & - & - & - \\
\hline 13 & Achrechne racemose & + & - & - & - & - & - & - & - & - \\
\hline 14 & Aristida adscensionis L. & + & - & - & - & - & - & - & - & - \\
\hline 15 & Aristida cyanantha Nees ex Steud & + & - & - & - & - & - & - & - & - \\
\hline
\end{tabular}




\begin{tabular}{|c|c|c|c|c|c|c|c|c|c|c|}
\hline 16 & Argyrolobium roseum & + & - & - & - & - & - & - & - & - \\
\hline 17 & Avena fatua Linn. & + & - & - & - & - & - & - & - & - \\
\hline 18 & Arndu dedonix & + & - & - & - & - & - & - & - & - \\
\hline 19 & Brachiaria ramose & + & - & - & - & - & - & - & - & - \\
\hline 20 & Brachiaia reptans & + & - & - & - & - & - & - & - & - \\
\hline 21 & Bromus japonica Thunb. & + & - & - & - & - & - & - & - & - \\
\hline 22 & Cymbopogon jwarancusa (Jones) Schult. & + & - & + & - & - & - & - & - & - \\
\hline 23 & Cynodon dactylon (L.) Pers. & + & - & + & - & - & - & - & - & - \\
\hline 24 & Cenchrus ciliaris L. & + & - & - & - & - & - & - & - & - \\
\hline 25 & Dinebra retroflexa & - & + & - & - & - & - & - & - & - \\
\hline 26 & Disteria cilians & - & + & - & - & - & - & - & - & - \\
\hline 27 & Desmostachya bipinnata (L.) Stapf. & - & + & + & - & - & - & - & - & - \\
\hline 28 & Dactyloctenium carstatum & + & - & - & - & - & - & - & - & - \\
\hline 29 & Dactyloctenium aegyptium (L.) Willd. & + & - & - & - & - & - & - & - & - \\
\hline 30 & Dichanthium annulatum (Forssk.) Stapf & + & - & - & - & - & - & - & - & - \\
\hline 31 & Eragrostis minor L. & + & + & - & - & - & - & - & - & - \\
\hline 32 & Echinochloa colona (Linn.) Link & + & - & - & - & - & - & - & - & - \\
\hline 33 & Hordeum vulgare L. & + & - & - & - & - & - & - & - & - \\
\hline 34 & Imperata cylindrica (Linn.) Raeuschel & + & - & - & - & - & - & - & - & - \\
\hline 35 & Lolium temulentum Linn. & + & - & - & - & - & - & - & - & - \\
\hline 36 & Oryza sativa $L$. & + & + & - & - & - & - & - & - & - \\
\hline 37 & Polypogon monspeliensis (L.) Desf. & + & - & - & - & - & - & - & - & - \\
\hline 38 & Phragmites karka (Retz) Trin. & - & - & + & - & - & - & - & - & - \\
\hline 39 & Pennisetum oriantale & + & + & - & - & - & - & - & - & - \\
\hline 40 & Phalaris minor Retz. & + & - & - & - & - & - & - & - & - \\
\hline 41 & Poa annua Linn. & + & - & - & - & - & - & - & - & - \\
\hline 42 & Sorghum vulgare(L.) Pers. & + & - & - & - & - & - & - & - & - \\
\hline 43 & Setaria vercillata (L.) & - & + & - & - & - & - & - & - & - \\
\hline 44 & Saccharum bengalensis & + & - & - & - & - & - & - & - & - \\
\hline 45 & Triticum aestivum L. & + & + & - & - & - & - & - & - & - \\
\hline 46 & Zea mays L. & + & - & - & - & - & - & - & - & - \\
\hline \multicolumn{11}{|c|}{ 10. Typhaceae } \\
\hline 47 & Typha latifolia L. & + & - & - & - & - & - & - & - & - \\
\hline $\begin{array}{l}\text { Ang } \\
11 .\end{array}$ & $\begin{array}{l}\text { sperm } \\
\text { canthaceae }\end{array}$ & & & & & & & & & \\
\hline
\end{tabular}




\begin{tabular}{|c|c|c|c|c|c|c|c|c|c|c|}
\hline 48 & Dicleptera bupleuroides & - & + & - & - & - & - & - & - & - \\
\hline \multicolumn{11}{|c|}{ 12. Aizoaceae } \\
\hline 49 & Zelays petendra (L.) C. Jeffery & - & - & - & - & - & - & - & - & - \\
\hline 50 & Trianthema portulacastrum L. & + & - & - & - & - & - & - & - & - \\
\hline \multicolumn{11}{|c|}{ 13. Amaranthaceae } \\
\hline 51 & Achyranthus aspera L. & - & + & - & - & - & - & - & - & - \\
\hline 52 & Achyranthus biclentata & - & + & - & - & - & - & - & - & - \\
\hline 53 & Amaranthus viridis L. & + & + & - & + & - & - & - & - & - \\
\hline 54 & Aerva javanica (Burn.f.) Juss & + & - & - & - & - & - & - & - & - \\
\hline 55 & Celosia cristata & + & + & - & - & - & - & - & - & - \\
\hline 56 & Digera muricata & + & - & - & - & - & - & - & - & - \\
\hline \multicolumn{11}{|c|}{ 14. Anacardaceae } \\
\hline 57 & Mengifera indica & + & + & + & - & + & - & - & - & - \\
\hline \multicolumn{11}{|c|}{ 15. Apiaceae } \\
\hline 58 & Coriandrium sativum L. & + & + & - & + & - & - & - & - & - \\
\hline 59 & Foeniculum valgare Mill. & - & + & - & - & - & - & - & - & - \\
\hline 60 & Scandix pectinvenens & - & + & - & - & - & - & - & - & - \\
\hline \multicolumn{11}{|c|}{ 16. Apocyanaceae } \\
\hline 61 & Rhazya stratica Decne. & - & + & + & - & - & - & - & - & - \\
\hline \multicolumn{11}{|c|}{ 17. Asclepiadaceae } \\
\hline 62 & Caralluma tuberculata N.E. Brown & + & + & - & + & - & - & - & - & - \\
\hline 63 & Calotropis procera (Wild) R.Br. & - & - & - & - & - & - & - & - & - \\
\hline 64 & Calotropis Gigantea L. R. Br. & - & - & - & - & - & - & - & - & - \\
\hline 65 & Periploca aphylla Decne. & + & + & + & - & - & - & - & - & - \\
\hline \multicolumn{11}{|c|}{ 18. Asteraceae } \\
\hline 66 & Artemisia scoparaia. Waldst \& Kitam & + & + & - & - & - & - & - & - & - \\
\hline 67 & Carthamus oxycantha Bieb & - & + & - & - & - & - & - & - & - \\
\hline 68 & Conyza bonariensis & - & - & - & - & - & - & - & - & - \\
\hline 69 & Conyza canadensis (L.) Cronquist & + & - & - & - & - & - & - & - & - \\
\hline 70 & Dendrathema indicum & - & + & - & - & - & - & - & - & - \\
\hline 71 & Filago hunclwarica & + & - & - & - & - & - & - & - & - \\
\hline 72 & $\begin{array}{l}\text { Filago arenaria (Smoljan.) Chrtek \& } \\
\text { Holub }\end{array}$ & + & - & - & - & - & - & - & - & - \\
\hline 73 & Helianthus annus L. & + & + & - & - & - & - & - & - & - \\
\hline 74 & Iphiona scabra DC.k & + & - & - & - & - & - & - & - & - \\
\hline
\end{tabular}




\begin{tabular}{|c|c|c|c|c|c|c|c|c|c|c|}
\hline 75 & Lactuca serriola L. & + & + & - & + & - & - & - & - & - \\
\hline 76 & Lactuca sativa L. & + & - & - & + & - & - & - & - & - \\
\hline 77 & Launaea procumbens Roxb. & + & - & - & - & - & - & - & - & - \\
\hline 78 & Leaucaena nudicaulis (L.) Hook. & + & - & - & - & - & - & - & - & - \\
\hline 79 & Pulicaria crispa (Forssk.) Oliv. & - & - & - & - & - & - & - & - & - \\
\hline 80 & Parthenium hysterophous L. & - & - & - & - & - & - & - & - & - \\
\hline 81 & Sonchus asper (L.) Hill. & + & + & - & - & - & - & - & - & - \\
\hline 82 & Sonchus oleraceus & + & + & - & - & - & - & - & - & - \\
\hline 83 & Saussurea heteromalla (D.Don.)Hand & + & - & - & - & - & - & - & - & - \\
\hline 84 & Taraxacum officinale Weber & + & - & - & - & - & - & - & - & - \\
\hline 85 & Xanthium strumarium L. & - & - & - & - & - & - & - & - & - \\
\hline \multicolumn{11}{|c|}{ 19. Boraginaceae } \\
\hline 86 & Cordia maxia Linn. & + & + & - & - & - & - & - & - & - \\
\hline 87 & Cynoglossum lanceolatum Forssk & + & - & - & - & - & - & - & - & - \\
\hline 88 & Ehrctia obtusifolia & + & - & - & - & - & - & - & - & - \\
\hline 89 & Heliotropium europaeum L. & + & + & - & - & - & - & - & - & - \\
\hline 90 & Heliotropism elipticum Ledeb. & + & + & - & - & - & - & - & - & - \\
\hline \multicolumn{11}{|c|}{ 20. Brassicaceae } \\
\hline 91 & Arabidoptis thaliana & + & - & - & - & - & - & - & - & - \\
\hline 92 & Brassica rapa $\mathrm{L}$. & + & - & - & - & - & - & - & - & - \\
\hline 93 & Brassica olaraceae L. & + & - & - & + & - & - & - & - & - \\
\hline 94 & Brassica campestris & + & - & - & + & - & - & - & - & - \\
\hline 95 & Coronopus didymus (L) Smith. & + & - & - & - & - & - & - & - & - \\
\hline 96 & Lepidium draba & + & - & - & - & - & - & - & - & - \\
\hline 97 & Raphanus sativus L. & + & + & - & + & - & - & - & - & - \\
\hline 98 & Sissymbrium irrio L. & + & + & - & - & - & - & - & - & - \\
\hline \multicolumn{11}{|c|}{ 21. Buxaceae } \\
\hline 99 & Buxus wallichiana Bail & + & - & - & - & - & - & - & - & - \\
\hline \multicolumn{11}{|c|}{ 22. Capparidiaceae } \\
\hline 100 & Capparis spinosa L. & + & + & + & - & - & - & - & - & - \\
\hline \multicolumn{11}{|c|}{ 23. Cleomaceae } \\
\hline 101 & Cleome brachycarpa Vahl ex DC. & - & - & - & - & - & - & - & - & - \\
\hline \multicolumn{2}{|c|}{ 24. Caryophyllaceae } & - & + & - & - & - & - & - & - & - \\
\hline 102 & Saponaria vaccaria & - & + & - & - & - & - & - & - & - \\
\hline 103 & Stellaria media L. Vill & - & + & - & - & - & - & - & - & - \\
\hline
\end{tabular}




\begin{tabular}{|c|c|c|c|c|c|c|c|c|c|c|}
\hline \multicolumn{11}{|c|}{ 25. Cannabinaceae } \\
\hline 104 & Cannabis sativus L. & - & + & - & - & - & - & - & - & - \\
\hline \multicolumn{11}{|c|}{ 26. Chenopodiaceae } \\
\hline 105 & Chenopodium album $\mathrm{L}$ & + & + & - & + & - & - & - & - & - \\
\hline 106 & Chenopodium murale L. & + & + & - & + & - & - & - & - & - \\
\hline 107 & Chenopodium nepalense Colla & + & + & - & + & - & - & - & - & - \\
\hline 108 & Chenopodium ambrosioides L. & + & + & - & + & - & - & - & - & - \\
\hline 109 & Suaeda fruticosa Forssk. Ex J.F. Gmelin & + & + & - & - & - & - & - & - & - \\
\hline \multicolumn{11}{|c|}{ 27. Convolvulaceae } \\
\hline 110 & Convunvulus arvensis $\mathrm{L}$. & + & + & - & - & - & - & - & - & - \\
\hline \multicolumn{11}{|c|}{ 28. Cucurbitaceae } \\
\hline 111 & Cucumis sativus Linn. & - & + & - & + & - & - & - & - & - \\
\hline 112 & Cacumis propheterum & - & - & - & - & - & - & - & - & - \\
\hline 113 & Cucurbita pepo L. & - & + & - & + & - & - & - & - & - \\
\hline 114 & Cucurbita maxima Duchesne. & - & + & - & + & - & - & - & - & - \\
\hline 115 & Legenaria siceraria & + & - & - & - & - & - & - & - & - \\
\hline \multicolumn{11}{|c|}{ 29. Cuscutaceae } \\
\hline 116 & Cuscuta reflexa Roxb & & + & - & - & - & - & - & - & - \\
\hline \multicolumn{11}{|c|}{ 30. Euphorbiaceae } \\
\hline 117 & Euphorbia hirta & + & + & - & - & - & - & - & - & - \\
\hline 118 & Euphorbia helioscopia L. & - & + & - & - & - & - & - & - & - \\
\hline 119 & Euphorbia prostrate Ait. & + & + & - & - & - & - & - & - & - \\
\hline 120 & Echinops echinatus D.C & + & - & - & - & - & - & - & - & - \\
\hline 121 & Ricinus communis L. & - & + & - & - & - & - & - & - & - \\
\hline \multicolumn{11}{|c|}{ 31. Fumaraceae } \\
\hline 122 & Fumariai ndica (Hausskn.) Pugsley & + & - & - & - & - & - & - & - & - \\
\hline \multicolumn{11}{|c|}{ 32. Lamiaceae } \\
\hline 123 & Salvia aegyptiaca Wall & - & + & - & - & - & - & - & - & - \\
\hline 124 & Mentha longifolia & - & + & - & + & - & - & - & - & - \\
\hline 125 & Mentha arvensis L. & - & + & - & + & - & - & - & - & - \\
\hline 126 & Ocimum bascillicum L. & - & + & - & - & - & - & - & - & - \\
\hline \multicolumn{11}{|c|}{ 33. Malvaceae } \\
\hline 127 & Abelmoschus esculentus (L.) & - & - & - & + & - & - & - & - & - \\
\hline 128 & Hibiscus trionum & - & + & - & - & - & - & - & - & - \\
\hline
\end{tabular}




\begin{tabular}{|c|c|c|c|c|c|c|c|c|c|c|}
\hline 129 & $\begin{array}{l}\text { Malvastrum coromandelianum (L.) } \\
\text { Gareke. }\end{array}$ & + & + & + & - & - & - & - & - & - \\
\hline \multicolumn{11}{|c|}{ 34. Meliaceae } \\
\hline 130 & Melia azedarach L. & - & + & + & - & - & - & - & - & - \\
\hline \multicolumn{11}{|c|}{ 35. Mimosaceae } \\
\hline 131 & Acacia nilotica (L) Delice. & + & + & + & - & - & + & - & + & - \\
\hline 132 & Acacia modesta Wall. & + & + & + & - & - & + & + & + & - \\
\hline 133 & Albizia lebbeck (L.) Benth. & + & + & + & - & - & + & + & + & - \\
\hline \multicolumn{11}{|c|}{ 36. Moraceae } \\
\hline 134 & Ficus palmate & + & + & + & - & + & - & - & - & - \\
\hline 135 & Morus alba L. & + & + & + & - & + & - & - & - & - \\
\hline 136 & Morus nigra L. & + & + & - & - & - & - & - & - & - \\
\hline \multicolumn{11}{|c|}{ 37. Myrtaceae } \\
\hline 137 & Eucalyptus globules L. & + & + & + & - & - & + & - & - & - \\
\hline 138 & Eucalyptus lanceolatus & + & + & + & - & - & + & - & - & - \\
\hline 139 & Syzygium cuminii (L.) Skeels & + & + & - & - & - & - & - & - & - \\
\hline \multicolumn{11}{|c|}{ 38. Musaceae } \\
\hline 140 & Musa paradisiaca L. & + & + & - & - & + & - & - & - & - \\
\hline \multicolumn{11}{|c|}{ 39. Nyctaginaceae } \\
\hline 141 & Mirabilis jalapa L. & - & - & - & - & - & - & - & - & - \\
\hline \multicolumn{11}{|c|}{ 40. Papilionaceae } \\
\hline 142 & Astragalus psilocentros Fisch. & + & + & + & - & - & - & - & - & - \\
\hline 143 & Cicer arietinum $\mathrm{L}$. & + & - & - & - & - & - & - & - & - \\
\hline 144 & Dalbergia sissoo Roxb. & + & + & + & - & - & + & + & - & + \\
\hline 145 & Medicago laciniata (L.) Mill. & + & - & - & - & - & - & - & - & - \\
\hline 146 & Melilotus longifolia (L.) & - & + & - & - & - & - & - & - & - \\
\hline 147 & Melilotus indicus (L.) All. & - & + & - & - & - & - & - & - & - \\
\hline 148 & Trigonella mcisa & + & - & - & - & - & - & - & - & - \\
\hline 149 & Vicia sativa $L$. & + & - & - & - & - & - & - & - & - \\
\hline \multicolumn{11}{|c|}{ 41. Papaveraceae } \\
\hline 150 & Papaver somniferum & + & + & - & - & + & - & - & - & - \\
\hline \multicolumn{11}{|c|}{ 42. Plantaginaceae } \\
\hline 151 & Plantago lanceolata L. & + & - & - & - & - & - & - & - & - \\
\hline 152 & Plantago minor L. & + & - & - & - & - & - & - & - & - \\
\hline
\end{tabular}




\begin{tabular}{|c|c|c|c|c|c|c|c|c|c|c|}
\hline 153 & Portulaca quardrifida $L$. & + & + & - & - & - & - & - & - & - \\
\hline \multicolumn{11}{|c|}{ 44. Polygonaceae } \\
\hline 154 & Calligonum polygonoides L. & + & + & + & - & - & - & - & - & - \\
\hline 155 & Polygonum plebeium $\mathrm{R} . \mathrm{Br}$ & + & - & - & - & - & - & - & - & - \\
\hline 156 & Rumex dentatusL. & + & + & - & - & - & - & - & - & - \\
\hline 157 & Rumex hastatus & + & + & - & - & - & - & - & - & - \\
\hline 158 & Rumex vesicarius & + & + & - & - & - & - & - & - & - \\
\hline \multicolumn{11}{|c|}{ 45. Primulaceae } \\
\hline 159 & Anagallis arvensis & + & - & - & + & - & - & - & - & - \\
\hline \multicolumn{11}{|c|}{ 46. Punicaceae } \\
\hline 160 & Punica granatum L. & + & + & + & - & + & - & - & - & - \\
\hline \multicolumn{11}{|c|}{ 47. Ranunculaceae } \\
\hline 161 & Ranunculus muricatus L. & - & + & - & - & - & - & - & - & - \\
\hline \multicolumn{11}{|c|}{ 48. Resedaceae } \\
\hline 162 & Reseda odorata L. & + & - & - & - & - & - & - & - & - \\
\hline 163 & Oligomeris linifolia (Vahl.) Macbride & - & - & - & - & - & - & - & - & - \\
\hline \multicolumn{11}{|c|}{ 49. Rosaceae } \\
\hline 164 & Rosa indica L. & + & + & - & - & - & - & - & - & - \\
\hline \multicolumn{11}{|c|}{ 50. Rhamnaceae } \\
\hline 165 & Zizyphus nummularia. (Burm.f) W.\&A & + & + & + & - & + & - & + & + & - \\
\hline 166 & Zizyphus oxyphylla Edge. & + & + & + & - & + & + & + & + & + \\
\hline 167 & Zizyphus mauritiana & + & + & + & - & + & + & + & + & + \\
\hline \multicolumn{11}{|c|}{ 51. Rubiaceae } \\
\hline 168 & Galium aparine & - & + & - & - & - & - & - & - & - \\
\hline 169 & Galium tricorne & - & + & - & - & - & - & - & - & - \\
\hline \multicolumn{11}{|c|}{ 52. Salvadoraceae } \\
\hline 170 & Salvadora oleoides Decne. & + & + & - & - & - & - & - & - & - \\
\hline \multicolumn{11}{|c|}{ 53. Salicaceae } \\
\hline 171 & Populus alba $\mathrm{L}$ & - & - & - & - & - & - & - & - & - \\
\hline \multicolumn{11}{|c|}{ 54. Sapindaceae } \\
\hline 172 & Dodonaea viscosa $L$. & - & + & + & - & - & - & - & - & - \\
\hline \multicolumn{11}{|c|}{ 55. Sapotaceae } \\
\hline 173 & Monotheca buxifolia (Falc.) A. DC. & + & - & - & - & - & - & - & - & - \\
\hline $56 . S$ & rophulariaceae & & & & & & & & & \\
\hline
\end{tabular}




\begin{tabular}{|c|c|c|c|c|c|c|c|c|c|c|}
\hline 174 & Veronica aquatica Bern & - & - & - & - & - & - & - & - & - \\
\hline 175 & Lindenbergia indica & - & - & - & - & - & - & - & - & - \\
\hline 176 & Kickxia incana (Wall) Penn. & - & + & - & - & - & - & - & - & - \\
\hline \multicolumn{11}{|c|}{ 57. Solanaceae } \\
\hline 177 & Capsicum annum L. & - & + & - & + & - & - & - & - & - \\
\hline 178 & Datura alba Nees & - & + & - & - & - & - & - & - & - \\
\hline 179 & Datura innoxia & - & + & - & - & - & - & - & - & - \\
\hline 180 & Hyosyamus niger & - & + & - & - & - & - & - & - & - \\
\hline 181 & Hyoscyamus squarrosus Griffith. & - & - & - & - & - & - & - & - & - \\
\hline 182 & Hyosyamus insanus & - & + & - & - & - & - & - & - & - \\
\hline 183 & Lycopersicom esculentum Mill. & + & - & - & + & - & - & - & - & - \\
\hline 184 & Solanum surattense Burm.f. & + & + & - & - & - & - & - & - & - \\
\hline 185 & Solanum melangena & + & + & - & + & - & - & - & - & - \\
\hline 186 & Solanum tuberosum & + & + & - & + & - & - & - & - & - \\
\hline 187 & Withania coagulans Dunal. & - & + & - & - & - & - & - & - & - \\
\hline 188 & Withania somnifera (L.) Dunal. & - & + & + & - & - & - & - & - & - \\
\hline \multicolumn{11}{|c|}{ 58. Tamaricaceae } \\
\hline 189 & Tamarix aphylla (L.) Karst. & + & + & + & - & - & + & + & - & + \\
\hline \multicolumn{11}{|c|}{ 59. Thymelaceae } \\
\hline 190 & Thymelaea passerine & - & - & - & - & - & - & - & - & - \\
\hline \multicolumn{11}{|c|}{ 60. Verbenaceae } \\
\hline 191 & Verbena hybrida Briq. & - & - & - & - & - & - & - & - & - \\
\hline 192 & Verbena officinalis & - & - & - & - & - & - & - & - & - \\
\hline 193 & Vitex negundo L. & + & + & - & - & - & - & - & - & - \\
\hline 194 & Phylla nodifloraL. (Greene) & - & - & + & - & - & - & - & - & - \\
\hline \multicolumn{11}{|c|}{ 61. Vitaceae } \\
\hline 195 & Vites vinifera L. & + & + & - & - & + & - & - & - & - \\
\hline \multicolumn{11}{|c|}{ 62. Zygophyllaceae } \\
\hline 196 & Fagonia cretica L. & + & + & - & - & - & - & - & - & - \\
\hline 197 & Peganum harmala L. & - & + & + & - & - & - & - & - & - \\
\hline \multirow[t]{2}{*}{198} & Tribulus terrestris L. & + & + & - & - & - & - & - & - & - \\
\hline & Total & $129(65 \%)$ & $108(55 \%)$ & $30(15 \%)$ & $25(13 \%)$ & $11(6 \%)$ & $10(5 \%)$ & $8(4 \%)$ & $7(3.5 \%)$ & $4(2 \%)$ \\
\hline
\end{tabular}




\section{Vegetable utilization}

25 plants were used as a vegetable in Darazinda which amount to $13 \%$. Abelmoschus esculentus, Allium cepa, Allium sativum, Amaranthus viridis, Anagallis arvensis, Brassica campestris, Brassica olaraceae, Caralluma tuberculata, Capsicum annum, Chenopodium album, Chenopodium murale, Coriandrium sativum, Cucumis sativus, Cucurbita maxima, Cucurbita pepo,Lactuca sativa, Lactuca serriola, Lycopersicom esculentum, Mentha arvensis, Raphanus sativus, Solanum melangena, Solanum tuberosumetc were used as vegetables. These vegetables are mostly cultivated near the homes and caffer their needs to some extent. Similar results were obtained by Sher et al. [20] who reported 36 vegetablespecies. Hazrat et al. [29], Sher et al. [20], Badshah and Hussain [30], Razaq et al. [31] and Durrani et al. [32] also reported similar results.

\section{Fruit yielding plants}

The results showed that $11(6 \%)$ out of 198 plants in the area were fruit yielding. Mengifera indica, Morus alba, Morus nigra, Musa paradisiaca, Papaver somniferum, Phoenix dactylifera, Punica granatum, Vites vinifera, Zizyphus oxyphylla, Zizyphus mauritiana, Zizyphus nummularia were fruit yielding plants. Most of these first plants are cultivated, while some e.g Zizyphus nummularia etc were wild. Similar results were shown by Lubna et al. [33] studied that $4.8 \%$ area in Pakistan covered by forests. Ibrar et al. [15] studied Ranyal Hill, Shangla and documented 18 edible species. Sher and Al-Yemni [26] worked on higher altitude of Malam Jaba Swat and reported 14 wild fruit species.

\section{Timber wood utilization}

$10(5 \%)$ plants were used as a timber in this area. Acacia nilotica, Acacia modesta, Albizia lebbeck, Dalbergia sissoo, Eucalyptus globules, Eucalyptus lanceolatus, Phoenix dactylifera, Tamarix aphylla,
Zizyphus oxyphylla, Zizyphus mauritiana etc are used as timber wood for making doors and windows etc. Our result is similar with Sher et al. [20] who recorded 21 timber species from Chagharzai Valley, District Buner, Pakistan.

\section{Agricultural tool making}

As the area is backward and poor, peoples still use conventional agricultural techniques using modern agricultural tools. The local people have cultivated small farmlands with the help of indigenous agricultural tools. A total $4(2 \%)$ plants were used for making agriculture tools including Dalbergia sissoo, Tamarix aphylla, Zizyphus oxyphylla, Zizyphus mauritiana. Different wood species were used e. g wood of $Z$. mauritiana was used for making handles of axes and ploughs. Similar results were reported from different parts of the country by Sher and Al Yemni [26], Sher et al. [20], Durrani et al., [32], Ibrar et al. [15] and Zabihullah et al. [34]. Different wood species may differ in different parts of the country which are same uses. For ploughing the wood of $Z$. maurtiana is used for making handles of axes.

\section{Other utilization of plant resources}

Recent study showed that 07 (3.5\%) plant species were used for furniture making. Acacia modesta, Albizia lebbeck, Dalbergia sissoo, Phoenix dactylifera, Tamarix aphylla, Zizyphus oxyphylla, Zizyphus mauritiana, Zizyphus nummularia are used for making furniture. Also 07 (4\%) plants were used for fencing agricultural fields. Acacia nilotica, Acacia modesta, Albizia lebbeck, Phoenix dactylifera, Zizyphus oxyphylla, Zizyphus mauritiana, Zizyphus nummularia were used for fencing. Similar results were reported by Durrani et al. [32], Sher et al. [20], Sher and Al-yemni [26] reported similar results of plant uses for furniture from different areas of the country.

\section{Authors' contributions}

Design and conducted the experiment: $\mathrm{U}$ Samreen \& M Ibrar, Data collection, analysis 
and interpretation of the data: L Badshah, S Naveed \& I Khatak, Edited, reviewed and revised the paper: Imran.

\section{References}

1. Sher H \& Hussain F (2009). Ethnobotanical evaluation of some plant resources in Northern part of Pakistan. Afr J Biotechnol 8(17): 4066-4076.

2. Saima S, Dasti AA, Abbas Q \& Hussain F (2010). Floristic diversity during monsoon in Ayubia National Park District Abbottabad Pakistan. Pak J Pl Sci 16 (1): 43-50.)

3. Fazal H, Ahmad N, Rashid A \& Farooq S (2010). A Checklist of phanerogamic flora of Haripur Hazara, Khyber Pakhtunkhwa, Pakistan. Pak J Bot 42 (3): 1511-1522.

4. Nasrullah, Nisar M, Suliman \& Ali Z (2010). Ethnobotanical Wealth of Jandool Valley, Dir Lower, Khyber Pakhtunkhwa, Pakistan. International Journal of Phytomedicine 4: 351-354.

5. Barkatullah \& Ibrar M (2011). Plants profile of Malakand Pass Hills, District Malakand, Pakistan. Afr. J. Biotechnol. 10(73): 16521-16535.

6. Marwat S K, Rehman F, Usman K, Khakwani AA, Ghulam S, Anwar N, Sadiq M \& Khan SJ (2011). Medicoethnobotanical studies of edible wild fruit plants species from the flora of north western Pakistan (D. I. Khan district). JMPR 5(16): 3679-3686.

7. Naveed S, Hussain F, Khattak I \& Badshah L (2012). Floristic Composition and Ecological Characteristics of OleaAcacia Forest of Shamshokii District Karak. Global Journals Inc. 12(8): 3136.

8. Haq F (2012). The Ethno Botanical Uses of Medicinal Plants of Allai Valley, Western Himalaya Pakistan. International Journal of Plant Research 2(1): 21-34.

9. Alamgeer T, Ahmad, Rashid M, Malik MNH, Mushtaq MN, khan J, Qayyum R, Khan AQ, Muhammad N (2013). Ethnomedicinal Survey of plants of
Valley Alladand Dehri, Tehsil Batkhela, District Malakand, Pakistan. .IJBMSP 3(1): 23-32.

10. Khan $M$ \& Hussain $F$ (2013).Conservation status of plant species in Tehsil Takht-e-Nasrati, District Karak, Khyber PakhtunKhawa, Pakistan. Int. J Biodivers Conserv 5(1): 20-26.

11. Ahmad KS \& Habib S (2014). Indigenous Knowledge of Some Medicinal Plants of Himalaya Region, Dawarian Village, Neelum Valley, Azad Jammu and Kashmir, Pakistan. Universal Journal of Plant Science 2(2): 40-47.

12. Hussain A, Mirza SN, Khan IA \& Naeem MA (2009). Determination of relative species composition and seasonal plant communities of Nurpur reserved forest in scrub rangelands of District Chakwal. Pak J Agri Sci 46(1): 55-59.

13. Arshad M, Hussan AU, Ashraf MY, Noureen S \& Moazzam M (2008). Edaphicfactors and distribution of vegetation in the Cholistan desert, Pakistan. Pak J Bot 40(5): 1923-1931.

14. Nasir E \& Ali SI (1971-1995). Flora of Pakistan. Department of Botany University of Karachi.

15. Ali SI \& Qaiser M (1995-2007). Flora of Pakistan. Department of Botany University of Karachi.

16. Ibrar M, Hussain F \& Sultan A (2007). Ethnobotanical studies on plant resources of Ranyal Hills, District Shangla, Pakistan. Pak J Bot 39(2): 329-337.

17. Dilshad SMR, Rehman NU, Ahmad N \& Iqbal A (2010). Documentation of ethnoveterinary practices for mastitis in dairy animals in Pakistan. Pak Vet $J$ 30(3): 167-171.

18. Sardar AA \& Khan Z (2009). Ethnomedicinal studies on plant resources of Tehsil Shakargarh, District Narowal, Pakistan. Pak J Bot 41(1):1118.

19. Barkatullah, Ibrar M \& Hussain F (2009). Ethnobotanical studies of Charkotli Hills, 
Batkhela District, Malakand, Pakistan. Front. Biol. China 4(4): 539-548.

20. Sher Z, Khan ZD \& Hussain F 2011. Ethnobotanical studies of some plants of Chagharzai valley, District Buner, Pakistan. Pak J Bot 43(3): 1445-1452.

21. Ullah R, Hussain Z, Iqbal Z, Hussain J, Khan F, Khan N, Muhammad Z, Ayaz S, Ahmad S, Rehman N\& Hussain I (2010). Traditional uses of medicinal plants in Darra Adam Khel NWFP Pakistan. J. Med. Plants Res 4(17): 1815-1821.

22. Jan G, Khan MA, Gul F, Ahmad M, Jan M \& Zafar M (2010). Ethnobotanical study of common weeds of Dir Kohistan valley, Khyber Pakhtoonkhwa, Pakistan. Pak. J. Weed Sci Res 16(1): 81-88.

23. Mahmood A, Mahmood A \& Mahmood M (2012). Indigenous wild medicinal plants used by local people of Dudial area, District Mirpur, Azad Jammu and Kashmir, Pakistan. J Med Plants Res 6(13): 2686-2690.

24. Khan M, Musharaf S \& Shinwari ZK (2011). Ethnobotanical importance of halophytes of Noshpho salt mine, District Karak, Pakistan. Res Pharmaceut Biotechnol 3(4): 46-52.

25. Khan M \& Hussain F (2013). Conservation status of plant species in Tehsil Takht-e-Nasrati, District Karak, Khyber Pakhtun Khawa, Pakistan. Int J Biodivers Conserv 5(1): 20-26.

26. Bonet MA \& Valles J (2007). Ethnobotany of Montseny biosphere reserve (Catalonia, Iberian Peninsula): Plants used in veterinary medicine. $J$ Ethnopharmacol 110:130-147.

26. Sher H \& Al-Yemeni M (2011). Economically and Ecologically Important Plant Communities in High
Altitude Coniferous Forest of Malam Jabba, Swat, Pakistan. Saudi J Biol Sci 18(1): 53-61.

27. Ajaib M, Khan ZD, Khan N \& Wahab M (2010). Ethnobotanical studies on useful shrubs of District Kotli, Azad Jammu \& Kashmir, Pakistan. Pak J Bot 42(3): 1407-1415.

28. Deka D, Saikia P \& Konwer D (2007). Ranking of fuel wood species by Fuel Value Index. Energy Sources, part A. Recovery, Utilization, and Environmental Effects 29(16): 1499 1506.

29. Hazrat A, Nisar M, Shah J \& Ahmad S (2011). Ethnobotanical study of some elite plants belonging to Dir, Kohistan Valley, Khyber Pukhtunkhwa, Pakistan. Pak J Bot 43(2): 787-795.

30. Badshah L \& Hussain F (2011). Farmer preferences and use of local fodder flora in Tank District, Pakistan. Afr $J$ Biotechnol 10(32): 6062-6071.

31. Razaq A, Rashid A, Ali H, Ahmad H \& Islam M (2010). Ethnomedicinal potential of plants of Changa Vally district Shangla, Pakistan. Pak J Bot 42(5): 3463-3475.

32. Durrani MJ, Manzoor $M$ \& Irfan $S$ (2009). Folk uses of some plants of Quetta, Pakistan. Pak J Pl Sci 15(1): 1-9.

33. Lubna $H$ (2001). Analyzing Institutional Set-up of Forest Management in Pakistan, Jour. Pakistan Institute of Development Economics Res Report 182: 21-22.

34. Zabihullah Q, Rashid A \& Akhtar N (2006). Ethnobotanical survey of Kot Manzary Baba Valley, Malakand Agency, Pakistan. Pak J Plant Sci 12: 115-122. 